\title{
Determinación de la digestibilidad in vivo en ovinos utilizando dietas a base de forrajes tropicales
}

\author{
Liliana Valencia-Trujillo ${ }^{1}$, Jaime Restrepo-Paredes ${ }^{2}$, Danny Enrique \\ Cerón-Hernández ${ }^{3} \&$ Wilmer Ferney Herrera-García ${ }^{4}$ \\ ${ }^{1}$ truliva@gmail.com, ${ }^{2}$ jaimerestrepo@hotmail.com, ${ }^{3}$ dannyc521@yahoo.com, ${ }^{4}$ wilmerh@gmail.com \\ Escuela de Ciencias Agrícolas, Pecuarias y del Medio Ambiente. \\ Universidad Nacional Abierta y a Distancia. CEAD Palmira, Colombia.
}

\begin{abstract}
Resumen.- Realizamos una comparación de la digestibilidad in vivo de dos especies de forrajes de bajo valor nutritivo: el King Grass (Pennisetum hybridum) y el Resucitado (Malvaviscus arboreus Cav.). Determinamos la digestibilidad de los forrajes en cinco tratamientos con diferentes proporciones de las dos especies, mediante una prueba in vivo con 15 ovinos de la raza Africana. El forraje que mejores resultados presentó fue el $P$. hybridum y los tratamientos con diversas proporciones conteniendo esta especie. Esto se relaciona con una mayor biodisponibilidad de los principios nutritivos y el mayor porcentaje de nutrientes digestibles totales presentes en esta gramínea. A través de los resultados obtenidos se pretende generar información para la toma de decisiones sobre la conveniencia o no del cultivo de los forrajes mencionados por su consumo y aprovechamiento dentro de la dieta alimenticia en rumiantes.
\end{abstract}

Palabras clave: Análisis de Van Soest, Digestibilidad, King Grass, Nutrientes Digestibles Totales

Abstract.- We compared digestibility by testing five different proportions of two low-nourishing species: King Grass (Pennisetum hybridum) and the shrub known as "Resuscitado" (Malvaviscus arboreus Cav.). We fed those to 15 African male sheep, of approximately $20 \mathrm{~kg}$ and one year old. We determined the digestibility of the forages in five treatments through Anova analysis and a comparisonof the entire digestible nutrients (NDT) with Duncan's multiple range test. P. hybridum was the forage with better digestibility and the treatments with diverse portions of two forages including this species. Ithas agreater bioavailability of the main nutrients and the highest percentage of total digestible nutrients present in this grass. Our results are usefulto make decisions about whether or not to cultivate these forage speices to use them as food for ruminants.

Key words: Analysis of Van Soest, Digestibility, King Grass, Total Digestible Nutrients

\section{Introducción}

La producción animal en países tropicales como Colombia se caracteriza por marcadas fluctuaciones en la cantidad y calidad de las pasturas, que traen como consecuencia una baja productividad. Esto hace que sea necesario evaluar germoplasmas de forrajes adaptados y de buena calidad que provean mayores aportes nutricionales. Los forrajes tropicales se utilizan en mayor o menor proporción como fuente o alternativa de alimento para animales, según la capacidad del productor, a pesar de que su calidad nutritiva varía según la época del año y el periodo de corte.

En este trabajo del área de la nutrición animal, buscamos comparar la digestibilidad in vivo de dos especies de forraje con bajo valor nutritivo: la gramínea conocida como King Grass (Pennisetum hybridum) y una forrajera poco convencional conocida como Resucitado (Malvaviscus arboreus Cav.), que es una especie arbustiva perteneciente a la familia Malvaceae. A partir de esto determinamos la tasa de consumo voluntario de una combinación de 
estos dos tipos de forraje en diferentes proporciones, por parte de ovinos de la raza Africana. Este tipo de trabajos son importantes para la obtención de indicadores sobre los beneficios o perjuicios del uso de diferentes especies vegetales como forraje para alimentar grupos de animales piloto y además favorecen la toma de decisiones por parte de los productores de la cadena pecuaria.

Estudios realizados por el Centro Agronómico Tropical de Investigación y Enseñanza (CATIE) determinaron que el $M$. arboreus $y$ otras arbustivas como Cinodosculus acotinitolcos, $C$. chayamansa y Murus sp., son utilizadas en los sistemas agroforestales especializados para la producción animal intensiva, puesto que son forrajes de alto valor nutritivo cuando son suministrados en asociación con leguminosas y gramíneas y permiten reducir los aportes externos de abonos o fertilizantes nitrogenados. Estos forrajes pueden reemplazar completamente los concentrados fabricados con cereales y tortas de oleaginosas, sin reducir de la calidad ni la cantidad de leche producida y por tanto permiten niveles muy altos de intensificación, sin generar una dependencia de insumos externos (Benavides 1994).

López (1995) evaluó el potencial forrajero de $M$. arboreus en términos agroeconómicos y de respuesta animal para determinar los factores que influyen en la producción. Los resultados mostraron que un mayor tiempo entre podas produjo un ligero aumento en el contenido de materia seca en hojas y tallos tiernos. La frecuencia de poda no afectó significativamente el contenido de proteína cruda, aunque hubo un leve aumento al hacer podas cada tres meses. El efecto de la adición de diferentes niveles de estiércol sobre el contenido de proteína cruda en hojas y tallos tiernos no mostró diferencias significativas. $\mathrm{Al}$ usar $\mathrm{NH}_{4}-\mathrm{NO}_{3}$, los valores de proteína cruda y de digestibilidad de estos componentes fueron mayores que con el uso de estiércol (López 1995).

Por otra parte, se han realizado estudios sobre el uso de árboles en sistemas de producción con base en las problemáticas existentes, ya sean modelos de producción inapropiados, pérdida de cobertura boscosa, pérdida de fertilidad de los suelos, disminución de la riqueza de especies vegetales y animales, así como especies de macro y microorganismos que intervienen en la dinámica de reciclaje de los nutrientes en la relación planta-suelo que mantiene la fertilidad de los ecosistemas naturales (Gómez et al.1995). A partir de esto surgió la necesidad de buscar opciones productivas que llevaran implícita la conservación de los bosques, el suelo y el agua. Se evaluaron especies como $M$. arboreus, que ofrece un alto potencial productivo representado en biomasa de alta calidad y que puede ser cosechada durante las diferentes épocas del año (Gómez et al. 1995).

El pasto P. hybridum (King Grass) es un forraje ampliamente conocido y utilizado por los ganaderos en Colombia, debido a su alto rendimiento en volumen por hectárea cultivada. Sin embargo, debido a su bajo valor nutricional, se ha clasificado como de regular a deficiente y esta condición empeora a medida que el pasto es más viejo, causando problemas de desnutrición en el ganado. Su bajo valor nutricional se debe principalmente a la poca disponibilidad de carbohidratos, más que a su concentración. Este forraje posee un alto contenido de lignina que va aumentando con la edad y que empaqueta carbohidratos como la hemicelulosa y la celulosa, haciendo que el sistema digestivo de los rumiantes sea incapaz de degradarla, con la consecuente dificultad en la absorción de sustancias nutritivas (Flórez et al. 1998)

La digestibilidad de los nutrientes es la diferencia, expresada como un porcentaje, entre los nutrientes presentes en el alimento ingerido y la fracción correspondiente no digerida que aparece en la excreta. La importancia de determinar la digestibilidad de un alimento radica en que es un valor variable entre distintos alimentos y posee un valor práctico; una digestión incompleta frecuentemente representa pérdidas en la cadena productiva (Londoño 1993). Por medio de este estudio buscamos generar bases para la toma de decisiones sobre la conveniencia o inconveniencia de cultivar M. arboreus y P. hybridum para su consumo y aprovechamiento como parte de la dieta alimenticia suministrada en la cría de ovinos, mediante un experimento realizado en el municipio de Timbío en el centro del departamento del Cauca, Colombia, a $1760 \mathrm{~m}$ de altitud, con una temperatura promedio anual de $17^{\circ} \mathrm{C}$ y una precipitación de $2500 \mathrm{~mm}$ /año.

\section{Métodos}

Utilizamos 15 jaulas metabólicas elaboradas 
en forma artesanal con materiales de la región, en cada una de las cuales dispusimos un ovino macho de raza Africana de 12 meses de edad y un peso entre 19 y $20 \mathrm{~kg}$. Estos últimos fueron alimentados con forraje proveniente de las especies M. arboreus (Resucitado) y P. hybridum (King grass).

Como tratamientos experimentales utilizamos cinco combinaciones de forraje, con variaciones en el porcentaje de $M$. arboreus y P. hybridum (Tabla 1). Cada tratamiento fue suministrado a tres animales, los que significa que hubo tres repeticiones por tratamiento, donde cada animal representó una unidad experimental.

El trabajo de campo duró 20 días y fue dividido en dos periodos. El primer periodo se inició en el momento en que alojamos a los animales en un corral de acostumbramiento durante 12 días, periodo durante el cual los alimentamos con $M$. arboreus y $P$. hybridum, repartido en dos raciones, en la mañana y en la tarde, sin tener en cuenta los datos de consumo debido a que estos días fueron de adaptación de los animales a la nueva dieta. Durante este periodo los ovinos pudieron consumir agua a voluntad, sal mineralizada, bloque nutricional y complementos vitamínicos comer-ciales; además fueron desparasitados como preparación para el inicio del experimento.

El segundo periodo, llamado período de evaluación, inició en el día 13 cuando aplicamos un desparasitante por vía oral, luego de lo cual los animales fueron dejados en ayuno durante 24 horas con el fin de desocupar el rumen. En el día 14 introdujimos a los animales en las jaulas y suministramos la ración de acuerdo con los tratamientos mencionados (Tabla 1). Durante este periodo se pesó el alimento

Tabla 1. Porcentaje de cada especie de forraje en los tratamientos suministrados

\begin{tabular}{ccc}
\hline Tratamiento & M. arboreus & P. hybridum \\
\hline T1 & 100 & 0 \\
T2 & 0 & 100 \\
T3 & 75 & 25 \\
T4 & 50 & 50 \\
T5 & 25 & 75 \\
\hline
\end{tabular}

suministrado y la proporción de éste que fue rechazada por los animales. Los alimentos rechazados nunca fueron utilizados nuevamente. Tomamos muestras (75 g) del alimento ofrecido en los días 4, 5, 6 y 7 y se guardaron en un congelador para su posterior análisis. La tasa de consumo de forraje se determinó por la diferencia entre la cantidad de forraje suministrado y la cantidad de forraje rechazado por cada unidad experimental

Durante los siete días de evaluación realizamos una colección total de heces (CTH) (Lachmann \& Araujo 1999), medimos el peso total y se recogieron muestras representativas de las mismas (75g). Los días 4, 5, 6 y 7 pusimos a los ovinos unos chalecos para facilitar la recolección y evitar la contaminación con orina y otras sustancias, además del pisoteo por parte de los mismos animales. Dichas muestras fueron guardadas en un congelador, de la misma forma en que se había hecho con las muestras de alimento ofrecido.

A partir de las muestras congeladas, hicimos un análisis Van Soest completo, energía bruta $\mathrm{y}$ digestibilidad in vitro con las muestra de forraje; con las muestras de heces determinamos el contenido de materia seca, proteína, cenizas y energía bruta para determinar digestibilidad in vivo. Realizamos además la determinación de los principios nutritivos de $M$. arboreus y $P$. hybridum mediante un análisis bromatológico. Para el análisis estadístico, utilizamos un diseño completamente al azar, y un análisis de Varianza (ANAVA). Las diferencias significativas entre los tratamientos se determinaron por la prueba de rango múltiple de Duncan.

\section{Resultados}

Los resultados correspondientes al consumo de alimento, cantidad de excretas y nutrientes digestibles totales (NDT) para cada tratamiento y repetición se encuentran en la Tabla 2. El ANAVA indica que dentro de los tratamientos no hay variabilidad en la digestibilidad pero que entre tratamientos si se presentó una diferencia significativa (Tabla 3 ). La prueba de Duncan reveló diferencias significativas en cuanto a la digestibilidad. Las diferencias de NDT con respecto al P. hybridum (T2) fue en su orden: $6,95 \%$ para $\mathrm{T} 1,3,20 \% \mathrm{~T} 4,2,85 \%$ T5 y de $2,80 \%$ para T3.

Los valores encontrados en la prueba in vivo y 
Tabla 2. Resultados de las variables analizadas en el experimento

\begin{tabular}{lccccc}
\hline \multicolumn{1}{c}{ Variables } & T1 & T2 & T3 & T4 & T5 \\
\hline Número de ovinos & 3 & 3 & 3 & 3 & 3 \\
Período experimental(días) & 20 & 20 & 20 & 20 & 20 \\
Peso inicial ovinos (kg) & 19 & 20 & 19 & 20 & 20 \\
Consumo medio de alimento (kg/día) & 3.119 & 3.469 & 3.660 & 3.748 & 3.783 \\
Cantidad de excretas (g/día) & 324.43 & 378.77 & 319 & 344.33 & 512.66 \\
NDT & 67.85 & 74.80 & 72.0 & 71.60 & 71.95 \\
\hline
\end{tabular}

en el análisis in vitro son consistentes, pues los tratamientos que incluyen alguna proporción de $P$. hybridum en la ración, muestran mejor digestibilidad. El análisis de varianza para este parámetro muestra una diferencia significativa entre tratamientos. $P$. hybridum presenta mayor digestibilidad que $M$. arboreus. Cabe destacar que estos dos forrajes contribuyen a suplir los requerimientos en cuanto a proteína y energía, pero de igual modo se debe complementar ya que no alcanzan a cubrir el total de los requerimientos necesarios para la producción ovina.

El análisis bromatológico (Tabla 4) muestra a $M$. arboreus (T1) con el más alto porcentaje proteico con $20.97 \%$, seguido de T3 con $18.29 \%$, T4 con $15.62 \%$, T5 con $12.95 \%$ y por último T2 (100\% P. hybridum) con $10.28 \%$.

En cuanto a la fracción no nitrogenada, T1 (100\% P. hybridum) presentó el porcentaje más alto (58.94\%) seguido de T5 (56.09\%), T4 (53.24\%), T3 (50.38\%) y por último T1 ( $100 \%$ M. arboreus) con $47.54 \%$, estos valores teóricamente pueden suplir la energía necesaria de la dieta del ovino (50\%). La fibra tiene que ver con la digestibilidad del forraje y los resultados muestran el T2 (100\% P. hybridum) con $34.10 \%$, seguido de T5 (29.67\%), T4 (25.25\%), T3 (20.8\%) y por último T1 $(100 \%$ M. arboreus) con $16.40 \%$.

Luego de 20 días de experimentación, los animales alimentados con diferentes porcentajes de $P$. hybridum en la ración, obtuvieron mayores valores de digestibilidad que aquellos alimentadnos solo con $M$. arboreus, mostrando diferencias significativas entre los tratamientos propuestos en la investigación. El tratamiento con P. hybridum tuvo además una mayor digestibilidad medida en términos de NDT.

\section{Discusión y Conclusiones}

La mayor digestibilidad de $P$. hybridum está influenciada directamente por la composición química del mismo, la mayor biodisponibilidad de los principios nutritivos y el mayor

Tabla 3. ANAVA para digestibilidad (NDT) en los cinco tratamientos

\begin{tabular}{|c|c|c|c|c|c|c|}
\hline \multirow{2}{*}{ F.V. } & \multirow{2}{*}{ G.L. } & \multirow{2}{*}{ S.C. } & \multirow{2}{*}{ C.M. } & \multirow{2}{*}{ F.c. } & \multicolumn{2}{|c|}{ F.t. } \\
\hline & & & & & $5 \%$ & $1 \%$ \\
\hline Tratamientos $(t-1)$ & 4 & 73.73 & 18.4325 & 8.579 & 3.48 & $5.99^{* *}$ \\
\hline Error t $_{(\mathrm{r}-1)}$ & 10 & 21.485 & 2.1485 & & & \\
\hline TOTAL $_{(\mathrm{r}-1)}$ & 14 & 95.215 & & & & \\
\hline
\end{tabular}


Tabla 4. Análisis nutricional de los forrajes M. arboreus y P. hybridum (\%)

\begin{tabular}{cccccccccccc}
\hline Muestra & $\begin{array}{c}\text { M.S } \\
\text { Total }\end{array}$ & Prot. & E.E. & CEN & FDN & FDA & LDA & CHOS HEMI & CEL & $\begin{array}{c}\text { E.B. } \\
\text { Cal/gr }\end{array}$ \\
\hline Malvaviscus & 23.79 & 20.97 & 4.67 & 10.42 & 43.07 & 29.53 & 6.23 & 20.87 & 13.54 & 23.3 & 3.976 .42 \\
King grass & 22.56 & 10.28 & 1.28 & 11.5 & 68.71 & 45.39 & 9.54 & 8.23 & 23.32 & 35.89 & 3.910 .50 \\
\hline
\end{tabular}

Fuente: Lab. Nutrición Animal U. Nal. Palmira, 2007

porcentaje de NDT presentes en él. El mejor comportamiento del P. hybridum 100\% (T1) con respecto a la digestibilidad está influenciado positivamente por el porcentaje de NDT presentes en él en mayor concentración que los otros cuatro tratamientos.

Los resultados del análisis de la digestibilidad in vitro realizados en el Centro Internacional de Agricultura Tropical (CIAT), son consistentes con los análisis realizados a las muestras de las heces colectadas a los animales in vivo. Por medio de los porcentajes de digestibilidad podemos tomar decisiones en cuanto a cultivos de forrajes no convencionales que pueden formar parte de la dieta en la nutrición de rumiantes como los ovinos. Por otro lado, estas especies forrajeras realizan un aporte significativo al microclima, contribuyen a aumentar la oferta de agua superficial y captación de gases atmosféricos, resultando beneficiosas en la conservación y mantenimiento ambiental. No obstante y de acuerdo con Preston (1989) y Gutiérrez (2002), las evaluaciones de digestibilidad tienen gran importancia en la determinación de las propiedades nutricionales de los alimentos, es preciso indicar que por sí mismos no son suficientes para deducir las respuestas alimenticias de los animales. Incluso la toma de decisiones técnicas con base en este único dato puede conducir a situaciones contraproducentes.

Los resultados en esta investigación ameritan nuevos estudios de digestibilidad con respecto a los forrajes evaluados, tomando en consideración otros aspectos de gran importancia para una completa evaluación de los mismos. Sería conveniente determinar la digestibilidad del contenido nutricional completo de los forrajes (minerales, vitaminas, grasas, etc.) igualmente determinar la energía metabolizable y neta entre otros.

\section{Literatura citada}

Benavides, J. E. (Ed.). 1994. Árboles y Arbustos Forrajeros en América Central. Centro Agronómico Tropical de Investigación y Enseñanza, CATIE. Serie Técnica. Informe Técnico No. 236. $721 \mathrm{pp}$.

Flórez, O., Bolivar, D., Botero, J. \& Ibrahim, M. 1998. Parámetros nutricionales de algunas arbóreas leguminosas y no leguminosas con potencial forrajero para la suplementación de rumiantes en el trópico. Livestok Research for Rural Development. Vol 10 No. 1 pp. 16-21.

Gutiérrez, G. 2002. Evaluación de la digestibilidad "in vivo" de los forrajes brachiaria (Brachiaria decumbens), estrella (Cynodom lemfuensis) y kikuyo (Pennisetum clandestinum), producidos en la meseta de Popayán, en cobayos (Cavia porcellus). En Revista de investigación UNAD. Bogotá D.C.

Lachmann, M. \& O. Araujo Febres. 1999. La estimación de la digestibilidad en ensayos con rumiantes. Universidad de Zulia, Facultad de Ciencias Veterinarias. Maracaibo-Venezuela.

Gómez, M.E., L. Rodríguez, E. Murgueitio, C.I. Ríos, C.H. Molina, C.H. Molina, E. Molina y J.P. Molina. 1995. Árboles y arbustos forrajeros utilizados en alimentación animal como fuente protéica. CIPAV, Cali, Colombia. 129pp.

Londoño, H. F. 1993. Fundamentos de alimentación animal. Managua, Nicaragua.

López, Z., J. Benavides, M.J. Kass \& S. López. 1994. Utilización de la Amapola (Malvaviscus arboreus Cav.) en sistemas agroforestales con rumiantes menores. Taller Internacional sobre Sistemas Silvopastoriles en la Producción Ganadera. Matanzas, Cuba. p 23

Preston, R.L. 1989. Typical composition of feed for cattle and sheep. Feedstuffs 61:19

Recibido: 30 de junio de 2009 Aceptado: 15 de mayo de 2010 